\title{
FREIGHT TRAIN SCHEDULING WITH MINIMUM ENERGY CONSUMPTION
}

\author{
T.H. LE \& B. JAUMARD
}

Computer Science and Software Engineering Department, Concordia University, 1455 de Maisonneuve Blvd. West, Montreal, QC, Canada H3G1MB

\begin{abstract}
We consider energy-efficient scheduling of freight trains on a line with single-track sections, as is the case on many freight railways routes in North America. We determine the train speed that minimizes the energy consumption, taking into account the departure and arrival times and exploiting the waiting times. The proposed energy consumption model takes into account the ground topography, the speed, the number of axles the axle load and the type of locomotives. Some experiments are conducted in order to estimate the energy gain when adapting the train speed, using Canada Pacific Railway (CPR) data. Keywords: freight trains, optimization modeling
\end{abstract}

\section{INTRODUCTION}

Freight train, for its high energy efficiency and safety advantage compared to other land and air transportation options, plays a very important role in transportation in North America. In America, the annual cost for train industry is up to 9 billion USD [1]. This article studies the fuel consumption within the context of freight train schedule.

In order to deal with the fuel consumption, there are few main approaches, among them simulation is the more popular. Bai et al. [2] take into account the tractive power required and also the energy loss due to braking. Then by using simulation, they investigate several driving profiles, i.e. the way that trains should be driven (accelerating, coating, braking, etc.) so that the energy consumption is reduced. However, the simulation is done only in a limited environment of about $30 \mathrm{~km}$ long from Hefei and Chang'anji, China, and it is not clear how the train profiles behave in larger instances. Similarly in Chang and Morlok [3], the authors, using simulation, evaluate energy consumption with respect to different train driving profiles. The factors being considered are the tractive power, the tangents and the curves. In another vein, Howlett et al. [4] use control theory to derive the optimal switching points and consequently optimal driving strategies for freight trains. However, the article, as well as others taking the simulation approach, do not take into account the scheduling of the trains. This can be addressed with the optimization approach. Ghoseiri et al. [5] apply a simple formula to calculate the friction and deduce from it a formula for the fuel rate consumed by a train. The authors utilize the Pareto frontier method and formulate a non-linear programming model to deal with basic constraints such as headway (i.e. safety) constraints and deadlock constraints. However, these constraints are modelled using many binary variables which render the solution of the problem difficult and consequently, the size of data instances that can be solved is small, only a few trains and stations.

Our article addresses the problem of building train schedule in single-track railway while minimizing the fuel consumption. The article is organized as follows. First, we discuss factors that contribute to the train fuel consumption estimation and then a fuel consumption

This paper is part of the proceedings of the 15th International Conference on Railway Engineering Design and Operation (COMPRAIL)

www.witconferences.com 
formula; taking into account the train weight, train speed is derived. Next, a mathematical model is built, which aims to minimize the fuel consumption using the formula derived from the first step and satisfies the operational constraint. We then describe our two-phase method to solve the problem together with some numerical results.

\section{ESTIMATING FUEL CONSUMPTION}

\subsection{Assumptions}

In this project, we assume that the amount of fuel required to produce a unit of force is the same, regardless of the speeds of trains [5]. Therefore, we study the fuel assumption by studying the resistance of the trains.

The resistance of the train is composed of three components: train rolling resistance, grade resistance and curve resistance. Out of the three, train rolling resistance is the most important. The grade and curve resistances are both dependent only on the weight of the trains. For simplification, in this project we ignore the grade and the curve resistance as they do not depend on speed and we cannot control these two resistances by varying the speed. In the remaining part of this article, we discuss only train rolling resistance and subsequently the fuel consumption resulting from the rolling resistance.

\subsection{Train rolling resistance}

Train rolling resistance was studied by Davis in the 1920s and is still used today, e.g. in Refs [3, 5-7]. It takes the following form:

$$
\text { Train rolling resistance } R=A+B V+C V^{2} \text {, }
$$

where $R$ is train rolling resistance, $A$ component independent of train speed, $B$ component dependent on speed, $C$ drag coefficient based on the shape of the front of the train and other features affecting air turbulence and $V$ the train speed.

The fuel consumption estimation based on the rolling resistance can be derived as follows. Let $\mathrm{P}$ denote the horsepower produced by the locomotives of a given train. At constant speed, the fuel consumption per hour depends linearly on the horse power, according to [6]

$$
F=a+b P \quad g a l / h r
$$

Let $T E$ denote the tractive effort. We also have

$$
P=T E . V \quad \text { and } \quad F=a+b T E . V .
$$

Assuming the horsepower $T E$ is approximately equal to the train resistance $T E=R=\left(A+B V+C V^{2}\right)$. After some algebraic transformations and scaling, the fuel consumption takes the following form:

$$
E=\left(\frac{a^{\prime}}{V}+\left(A+B V+C V^{2}\right)\right) d \quad g a l
$$




\section{TRAIN SCHEDULING AND FUEL CONSUMPTION OPTIMIZATION MODEL}

\subsection{Generalities}

Although the energy consumption is very important in train operations, other aspect should be taken into account in order to maintain the quality of service. One of the other requirements is that train schedule should be reasonable with respect to the travelling time; i.e. the trains should not take too much time to travel from their origin to their final destination. The trains must, on the other hand, satisfy the operational constraints, e.g. safety constraints, no deadlock constraints [8]. These concerns are to some extent independent of the fuel consumption. Therefore, we propose a heuristic in which the optimization model is performed in two steps as follows.

The first step is to build a train schedule using the algorithm described in [8] which minimizes the average travelling time. The input of the optimization model includes the train departure times (which are planned by the train company), the speed limits (which implicitly take into account the network features such as grade, curvature, etc.), the number of siding tracks, etc. The schedule produced by the model will specify the arrival and departure of each train at each station while satisfying the operational constraints as specified in [8]. In other words, this first step builds an initial feasible schedule that minimizes the average travelling time.

In the second step, a more detailed schedule is built based on the schedule obtained from the first step. In the detailed schedule, the arrival and the departure times of the trains at each station are kept the same as the ones from the first step but the speed of each train in each part of its initiatory is determined by the mathematical model so that the fuel consumption of trains is minimized. The fuel consumption to be minimized is calculated using eqns (1) and (4).

The advantage of this approach is that we can reuse the mathematical model in [8] which leads to a scalable train scheduling algorithm. This model is also quite flexible and we can easily incorporate many types of operational constraints. Another important advantage is that in the first step, we work with all the trains, ensuring they have to satisfy all the operational constraints whereas in the second step, we work with each train individually and also each segment, finding a detailed schedule that minimizes its fuel consumption. This helps increasing significantly the scalability when solving the model.

As an output of the optimization model, we get the average speed in the segments, connecting two successive sidings/stations $p$ and $p^{\prime}$. In order to compute the energy consumption in that segment $\left[p, p^{\prime}\right]$, we may need to divide $\left[p, p^{\prime}\right]$ into sub-segments, on which the speed is constant. We next discuss how to do it on a base case that is depicted in Fig. 1. Generalization to more sub-segments is straightforward.

\subsection{Outline of energy consumption optimization in a segment}

Let I be the set of indices indicating the sub-segments. Let $v_{i}$ be the speed of train $\mathrm{t}$ on $\ell_{i}$, and $v_{\mathrm{opt}}$ the optimized average speed of the train between $\mathrm{p}$ and $\mathrm{p}^{\prime}$, as output by the optimization model.

$$
\min \sum_{i \in I}\left(\frac{a^{\prime}}{v_{i}}+A+B v_{i}+C v_{i}^{2}\right) \ell_{i}
$$




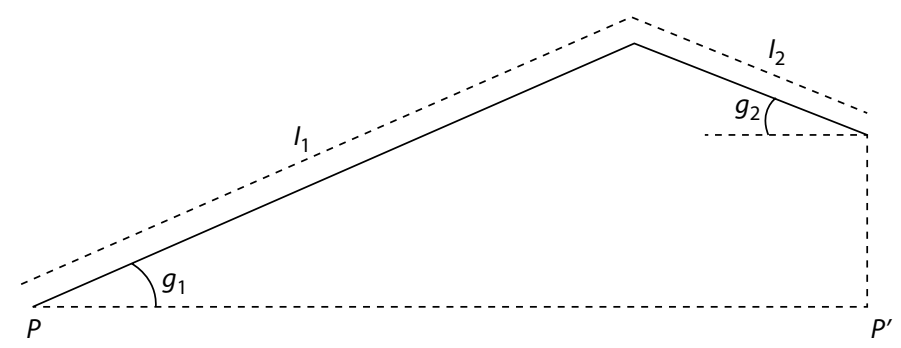

Figure 1: The case of two grade distributions in one segment.

$$
\begin{array}{cl}
\text { subject to: } & \sum_{i \in I} \frac{\ell_{i}}{v_{i}}=a_{t}^{p^{\prime}}-d_{t}^{p} \quad i \in I, \\
& v_{i} \in \mathbb{R} \quad i \in I
\end{array}
$$

In terms of solving techniques, the model above is in a separable form with respect to variables $v_{i}$, i.e. the objective and the left-hand side of the constraints are in the form of $\sum_{i \in I} f_{i}\left(v_{i}\right)$. Therefore, we can linearize the terms in the objective and the constraints, namely $E_{i}=\left(\frac{a^{\prime}}{v_{i}}+A+B v_{i}+C v_{i}^{2}\right) \ell_{i}$ and $\frac{\ell_{i}}{v_{i}}$. Moreover, they are all convex and we are minimizing the objective; therefore when we linearize using $\lambda$-method, the resulting linearization leads to a linear programming problem.

\subsection{Time domain formulation}

The formulation above with respect to speed has one disadvantage, that is the constraint (6) is non-linear and we need to linearize it. However, while linearizing a constraint, we could face infeasibility unless we linearize $v_{i}$ with a sufficiently small granularity. Then, however, the number of variables in the resulted linearization problem might become too large. One way to avoid this issue is, instead of modelling the problem using velocity variables $v_{i}$, we can use time index variables to reformulate the problem.

Let $t_{i}$ be the time it takes to travel $l_{i}$ then $v_{i}=\frac{\ell_{i}}{t_{i}}$ and the model can be written as follows:

$$
\begin{gathered}
\min \sum_{i \in I}\left(\frac{a^{\prime}}{\ell_{i}} t_{i}+A+B \frac{\ell_{i}}{t_{i}}+C \frac{\ell_{i}^{2}}{t_{i}^{2}}\right) \ell_{i} \\
\text { subject to: } \sum_{i \in I} t_{i}=a_{t}^{p^{\prime}}-d_{t}^{p} \\
t_{i} \in \mathbb{R} \\
\quad i \in I .
\end{gathered}
$$


It is easy to verify that the function $E_{i}=\left(\frac{a^{\prime}}{l_{i}} t_{i}+A+B \frac{\ell_{i}}{t_{i}}+C \frac{\ell_{i}^{2}}{t_{i}^{2}}\right)$ is still convex and separable in $t_{i}$. Therefore, if we are to apply linearization using $\lambda$-method, the resulting formulation is still a linear programming problem.

Let $t_{i}$ be linearized by the set of $k_{i}$ points $\tau_{i}^{1}, \tau_{i}^{2}, \ldots, \tau_{i}^{k_{i}}$ with associated $\lambda$ variables $\lambda_{i}^{1}, \lambda_{i}^{2}, \ldots, \lambda_{i}^{k_{i}}$. Besides, let $\tau_{i}^{1}$ and $\tau_{i}^{k_{i}}$ be equal to lower bound and upper bound of $t_{i}$, respectively. We then have the following linear programming problem:

$$
\begin{gathered}
\min \sum_{i} \sum_{j=1}^{k_{i}} E_{i}\left(\tau_{i}^{j}\right) \lambda_{i}^{j} \ell_{i} \\
\text { subject to : } \sum_{i} t_{i}=a_{t}^{p^{\prime}}-d_{t}^{p} \\
t_{i}=\sum_{j=1}^{k_{i}} \tau_{i}^{j} \lambda_{i}^{j} \quad i \in I, \\
\sum_{j=1}^{k_{i}} \lambda_{i}^{j}=1 \quad i \in I, \\
\lambda_{i}^{j} \geq 0 \quad i \in I, j \in J .
\end{gathered}
$$

\subsection{Safety constraint}

Assuming that, from the schedule produced from the first phase, in segment $\left[p, p^{\prime}\right]$, train $m^{\prime}$ depart after $m$ but the time $m^{\prime}$ spend $\left[p, p^{\prime}\right]$ overlaps with that of $m$. In other words, there is a time that both $m$ and $m^{\prime}$ are on segment $\left[p, p^{\prime}\right]$. We need to make sure that $m$ and $m^{\prime}$ maintain the safety distance in all point $i$.

In general, in any given segment $\left[p, p^{\prime}\right]$ there might be $k$ trains $m_{1}, m_{2},,, m_{k}$ in which $m^{\prime}$ overlaps with $m_{2}$ and $m_{2}$ overlaps with $m_{3}$ and so on. If this is the case, we need to maintain the safety distance between $m_{i}$ and $m_{i+1}$ in segment $\left[p, p^{\prime}\right]$. Our experiments with the data show that $k$ usually takes the value of 2 .

Let the set of sub-segments ends of $\left[p, p^{\prime}\right]$ be indexed by point $i$. Since train $m$ cannot stop in the middle of a segment, departure and arrival times at each point $i$ inside the segment are equal; i.e. we only need to be concerned with the departure time at each point $i$. Denote by $d_{i}^{m}$ the departure time at point $i$ of train $m$. Let $M$ be the set of trains that overlaps $m_{1}, m_{2}, \ldots m_{k}$ at segment $\left[p, p^{\prime}\right]$; i.e. train $m_{j}$ overlaps train $m_{j+1}$. We then have the following model:

$$
\begin{gathered}
\min \sum_{m \in M} \sum_{i \in I}\left(\frac{a^{\prime}}{\ell_{i}} t_{m i}+A+B \frac{\ell_{i}}{t_{m i}}+C \frac{\ell_{i}^{2}}{t_{m i}^{2}}\right) \ell_{i} \\
\text { subject to : } \quad t_{m i}=d_{i+1}^{m}-d_{i}^{m} \quad m \in M, i \in I,
\end{gathered}
$$




$$
\begin{array}{cc}
\sum_{i} t_{m i}=a_{m}^{p^{\prime}}-d_{m}^{p} & m \in M, \\
d_{i}^{m_{j}} \geq d_{i}^{m_{j+1}}+\text { safety_distance } & i \in I, \\
d_{i}^{m}, t_{m i} \in \mathbb{R} & m \in M, i \in I .
\end{array}
$$

Observe that the safety distance is then ensured by constraint (19).

\subsection{Taking advantage of the waiting time in the second phase}

In the area where there are only single tracks, when two trains meet, one of them has to slow down and wait on the siding for the other train to pass. The train schedule built from the first phase specifies where trains meet and how much time trains have to wait. Figure 2 shows an example of a train meeting. Train 1 goes from station $\mathrm{C}$ to $\mathrm{D}$ to $\mathrm{E}$ and Train 2 in the opposite direction from station $\mathrm{E}$ to $\mathrm{D}$ to $\mathrm{C}$. They meet at $\mathrm{D}$ at $08: 12$ and Train 1 has to take the siding and wait from 08:10 to 08:15.

As described above, the second phase will find a refined schedule for the trains so that the overall fuel consumption is minimized. For a train that waits at a siding such as Train 1 in the example, the wait time can be exploited to reduce speed and therefore fuel consumption as follows. In the original schedule obtained from phase 1, Train 1 passes the station $\mathrm{C}$ at 08:05 , arrives at $\mathrm{D}$ at 08:10 and waits there until 08:15 then departs from $\mathrm{D}$ and arrives at $\mathrm{E}$ at 08:22. Train 2 passes $\mathrm{E}$ at 08:07, $\mathrm{D}$ at 08:12 and $\mathrm{C}$ at 08:17. We adjust the timeline of Train 1 such that the Train 1 passes $C$ at 08:05 (same as the original schedule) and arrives at $\mathrm{D}$ at 08:12 (the time Train 2 passes $\mathrm{D}$ ). Train 1 then departs from $\mathrm{D}$ at 08:12 and arrives at $\mathrm{E}$ at 08:22. The adjusted timeline of Train 1 is depicted in dash line in Fig. 2. The timeline of Train 2 is kept the same.

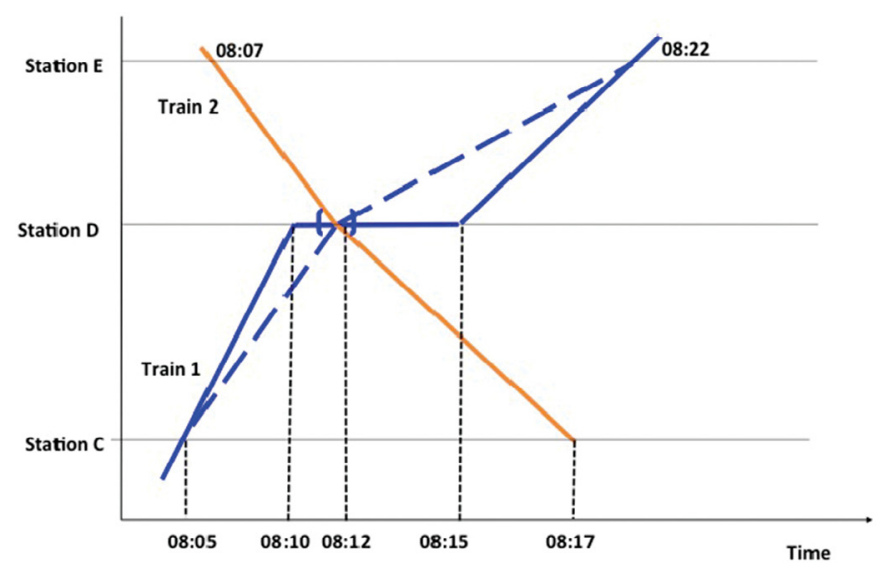

Figure 2: Adjust timeline of a waiting train. 
As can be seen, the adjustment above does not affect the feasibility of the schedule; therefore, in the second phase, the scheduling constraints will still be satisfied. Consequently, no binary variable is needed and the model in the second phase is a continuous linear problem and is highly scalable.

\subsection{Combining successive segments}

In addition to the improvement discussed in Section 3.5, it turns out that the schedule of the train can be adjusted so that the resistance and therefore fuel consumption can be further reduced. The schedule output in the first phase is optimized with respect to the travel time. Travelling times, i.e. the speed, may vary quite significantly from one segment to the next. So, if the optimization process is performed over several consecutive segments, then speed differences can be smoothed out and the overall saving could be larger than the combined savings on each individual segment. In that case, since the travelling time of each segment may be adjusted, the minimum travel time constraint in each segment should be added to the model so that the schedule is still feasible.

In principle, the more segments are combined, the more smoothness of the speeds between segments and therefore the more fuel consumption saving can be obtained. However, in order to maintain feasibility of the schedule, successive segments are combined, for any given train, until only it meets another train on opposite direction. For example, consider Train 2 in Fig. 3, travelling from station $\mathrm{F}$ going through $\mathrm{E}$ then meeting Train 1 at $\mathrm{D}$ and continuing its trip through $\mathrm{C}$ then $\mathrm{B}$ and $\mathrm{A}$ (not shown in Fig. 3). The successive segments that will be included into the set are F-E and E-D but not D-C. If segment D-C was included then the arrival and departure times of Train 2 at $\mathrm{D}$ would be modified and consequently Train 2 and Train 1 might no longer meet at D but inside either segment E-D or D-C and the schedule's feasibility no longer holds. The departure time of Train 2 at the first station of the segment set (F in this case) and the arrival time at the last one (D) will be fixed to those obtained from phase 1 (07:55 and 08:12 in Fig. 3). The departure and arrival at other stations are flexible, assuming the minimum travelling time constraint is satisfied.

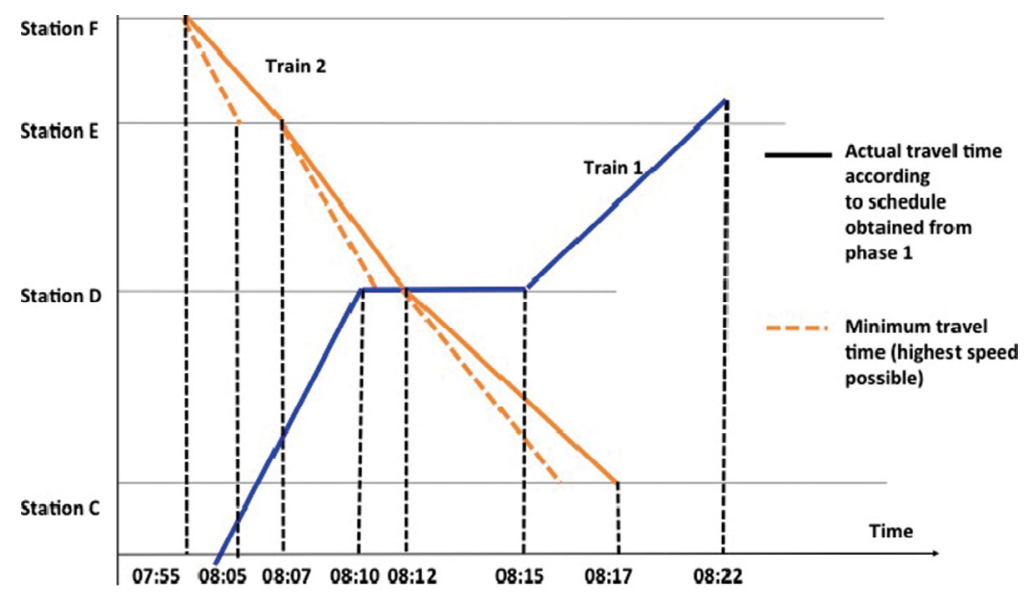

Figure 3: Combining consecutive segments of one train. 
Let $S$ be the set of segments, $i_{s}$ be the sub-segment index of segment $s, t_{s m i_{s}}$ be the time train $m$ spends at sub-segment $i_{s}$ of segment $s$ then the objective is modified as follows:

$$
\min \sum_{s \in S} \sum_{m \in M} \sum_{i \in I_{s}}\left(A+B \frac{\ell_{i_{s}}}{t_{s m i_{s}}}+C \frac{\ell_{i}^{2}}{t_{s m i_{s}}^{2}}\right) \ell_{i_{s}} .
$$

The following constraints must be satisfied in addition to the constraints (17)-(19):

$$
\begin{array}{cl}
t_{s m i_{s}}=d_{m}^{s}-d_{m}^{s} \geq \text { min_travel } & s \in S, m \in M, \\
\sum_{s \in S} t_{s m i_{s}}=a_{m}^{s^{\text {last }}}-d_{m}^{s^{\text {first }}} & m \in M, \\
d_{m}^{s}, t_{\text {smi }} \in \mathbb{R} & s \in S, i \in I .
\end{array}
$$

\section{NUMERICAL EXPERIMENTS}

As an initial step, we focus on the division Calgary to Field of the railway network of Canadian Pacific Railway. This part of the network consists essentially of single tracks with about 42 stations. We evaluate the energy consumption saving in 5 instances with a number of trains varying from 10 to 18 .

Table 1 shows the result of our experiments when we fixed the departure times as planned. The first and second columns correspond to the number of trains and their average travelling time obtained from phase 1 of the model. The third column is the fuel consumption obtained from the first phase without any optimization. This quantity is the sum of the consumption in each segment. To estimate each segment's consumption we assume the speed is constant and equal to the average speed in that segment, which can be deduced from the arrival and departure times obtained from the first phase. The fourth and fifth columns describe the fuel consumption and the percentage of the saving when we optimize the fuel consumption with the inclusion of waiting times. Similarly, the last two columns show the fuel consumption and the percentage of the saving in case we combine consecutive segments and include the waiting times. As can be seen from the last two columns of Table 1, the proposed approach shows significant saving of $14 \%$ to $16 \%$. Including waiting time in the optimization contributes the most to the reduction of energy consumption or about twothirds of this reduction; the remaining one-third of the saving comes from the combination of several segments.

Table 2 compares the two scenarios. In the first one, we fix the departure times of the trains as planned and in the second one, we allow the departure times to vary between 30 minutes before and 30 minutes after the planned departure time. Since the second scenario has more flexibility, it can reduce the average travelling time between 8 and 43 minutes depending on the number of trains. However, as the travelling time reduces, the fuel consumption tends to increase, as the last two columns of Table 2 show. Also, as can be seen in columns 6 and 9 of Table 2, the savings in case of fixed departure time are usually higher and vary much less than those in case of flexible departures. 
Table 1: Fuel consumption - fixed departure times.

\begin{tabular}{lcccccc}
\hline Train & $\begin{array}{c}\text { Average } \\
\text { travelling time }\end{array}$ & $\begin{array}{c}\text { Without } \\
\text { optimization }\end{array}$ & \multicolumn{3}{c}{$\begin{array}{c}\text { Fuel consumption (in gal) } \\
\text { With optimization }\end{array}$} \\
\hline & & & $\begin{array}{c}\text { Include wait } \\
\text { times }\end{array}$ & Saving & $\begin{array}{c}\text { Combine seg- } \\
\text { ments }\end{array}$ & Saving \\
& & & 22982.5 & $10.18 \%$ & 21909.1 & $14.38 \%$ \\
10 & $10: 18$ & 25587.9 & 26983.3 & $10.22 \%$ & 25483.2 & $15.21 \%$ \\
12 & $10: 19$ & 30055.7 & 30523.8 & $10.72 \%$ & 29140.5 & $14.77 \%$ \\
14 & $10: 28$ & 34188.8 & 34485.4 & $10.6 \%$ & 32363.7 & $16.1 \%$ \\
16 & $10: 28$ & 38572.3 & 37501.7 & $10.66 \%$ & 34960.8 & $16.72 \%$ \\
18 & $11: 02$ & 41977.7 & 372 & & &
\end{tabular}

Table 2: Fuel consumption - fixed vs flexible in departure time.

\begin{tabular}{|c|c|c|c|c|c|c|c|c|c|c|}
\hline \multirow[t]{3}{*}{ Train } & \multirow{2}{*}{\multicolumn{2}{|c|}{$\begin{array}{l}\text { Average } \\
\text { trav. time }\end{array}$}} & \multicolumn{8}{|c|}{ Fuel consumption (in gal) } \\
\hline & & & \multicolumn{3}{|c|}{ Fixed } & \multicolumn{3}{|c|}{ Flexible } & \multicolumn{2}{|c|}{$\begin{array}{c}\text { Diff. Fle \& } \\
\text { Fix }\end{array}$} \\
\hline & Fix & Fle & W/o opt. & . With opt. & Saving & W/o opt. & With opt. & Saving & (in gal) & $\%$ \\
\hline 10 & $10: 18$ & $9: 32$ & 25587.9 & 21909.1 & $14.4 \%$ & 26522.4 & 23792.2 & $10.29 \%$ & 1883.1 & 8.6 \\
\hline 12 & $10: 19$ & 10:11 & 30055.7 & 25483.2 & $15.2 \%$ & 30408.8 & 26739.2 & $12.07 \%$ & 1256 & 4.9 \\
\hline 14 & $10: 28$ & 10:08 & 34188.8 & 29140.5 & $14.8 \%$ & 34656.8 & 29067.8 & $16.13 \%$ & -72.7 & -0.2 \\
\hline 16 & $10: 28$ & $10: 13$ & 38572.3 & 32363.7 & $16.1 \%$ & 38743.9 & 33877.8 & $12.56 \%$ & 1514.1 & 4.7 \\
\hline 18 & 11:02 & $10: 19$ & 41977.7 & 34960.8 & $16.7 \%$ & 42324.8 & 36296.8 & $14.24 \%$ & 1336 & 3.8 \\
\hline
\end{tabular}

\section{CONCLUSION}

In this article, we develop a two-phase approach to address the problem of scheduling trains, satisfying different operational constraints while minimizing their fuel consumption. In the first phase, the train schedule is built with the objective of minimizing the travel time. In the second phase, the obtained schedule is optimized with respect to the fuel consumption. Some improvements are proposed so that the consumption is further minimized. Experiments are performed on reasonable size of real-life dataset of 42 stations and up to 18 trains with the savings between $10 \%$ and $16 \%$ depending on the instances. The results show that our method is scalable, and hence, could be used as a tool to evaluate different train scheduling scenarios.

\section{REFERENCES}

[1] Vantuono, W.C., A closer look at LNG. Railway Age, October 2013, p. 14.

[2] Bai, Y., Mao, Y., Zhou, F., Ding, Y. \& Dong, C., Energy-efficient driving strategy for freight trains based on power consumption analysis. Journal of Transportation, Systems Engineering and Information Technology, 9, pp. 43-50, 2009.

[3] Chang, D. \& Morlok, E., Vehicle speed profiles to minimize work and fuel consumption. Journal of Transportation Engineering, 131, pp. 173-182, 2005. 
[4] Howlett, P.G., Vu, X., Pudney, P.J., Local energy minimization in optimal train control. Automatica, 45, pp. 2692-2698, 2009.

[5] Ghoseiri, K., Szidarovszky, F. \& Asgharpour, M.J., A multi-objective train scheduling model and solution 2004. Transportation Research Part B: Methodological, 38(10), pp. 927-952, 2004.

[6] Radford, R.W., Fuel consumption of freight trains hauled by diesel electric locomotive. Journal of Engineering for Industry, 105, pp. 75-87, 1983.

[7] AREMA, American Railway Engineering and Maintenance-of-Way Association Manual, Maryland, USA, 2003.

[8] Jaumard, B., Le, T., Tian, H., Akgunduz, A. \& Finnie, P., A dynamic row/column management algorithm for freight train scheduling. 12th Workshop on Algorithmic Approaches for Transportation Modelling, Optimization, and Systems (ATMOS), Ljubljana, Slovenia, pp. 1-12, 2012. 